\title{
New Approaches for Studying the Chemical Diversity of Natural Resources and the Bioactivity of their Constituents
}

\author{
Jean-Luc Wolfender ${ }^{\star}$ and Emerson Ferreira Queiroz
}

\begin{abstract}
Natural products (NPs) have historically been an important source of lead molecules in drug discovery. However, the interest that the pharmaceutical industry has had in NPs has declined in part because of the lack of compatibility of traditional natural-product extract libraries with high-throughput screenings and the low hit rate. Furthermore, in contrast to the synthetic libraries, compounds from natural sources are likely to have complex structures which slow down the identification process and contribute to problems related to supply and manufacturing. In this paper, we summarise some of the strategies that are being developed in our research unit to address these issues. On one hand, differential screening strategies were established with the aim of identifying dynamically induced NPs from silent biosynthetic pathways in plants and fungi that had been exposed to different stress situations. On the other hand, high-resolution HPLC techniques were optimised for biological and chemical profiling of crude extracts. This led to an integrated platform for rapid and efficient identification of new drug-leads and biomarkers of interest that were based on miniaturised technological approaches and metabolomics.
\end{abstract}

Keywords: Bioactive natural products · Drug discovery · Metabolomics · Microflow NMR $\cdot$ Stress induction UHPLC-TOF-MS

\section{Introduction}

Through the natural selection process, natural products (NPs) possess a unique and vast chemical diversity that has evolved to form optimal interactions with biological macromolecules. ${ }^{[1]}$ NPs have thus inspired most of the active ingredients of medicines, and numerous NP 'scaffolds' have, over the years, led to a significant number of approved drugs and drug candidates to treat many diseases. ${ }^{[2]}$ The structural diversity of these compounds supports the belief that collections of NPs are not only more varied than those composed of synthetic compounds, but also that NPs better represent the 'chemical space' of drug-like molecules. ${ }^{[3]}$

Despite the great impact of naturally derived remedies, major pharmaceutical

${ }^{\star}$ Correspondence: Prof. J.-L. Wolfender Phytochimie et Produits Naturels Bioactifs Ecole de Pharmacie Genève-Lausanne Section des Sciences Pharmaceutiques Université de Genève

Quai Ansermet 30, $\mathrm{CH}-1211$ Genève 4

Tel.: +4122379 3385

Fax: +41223793399

E-mail: jean-luc.wolfender@unige.ch companies have scaled down their engagement in NP drug discovery during the last decade. ${ }^{[4]}$ According to Koehn and Carter, ${ }^{[5]}$ this could be attributed to different factors such as the introduction of high-throughput screening using specific molecular targets leading some companies to shift from natural products extract libraries to synthetic compounds libraries more appropriate to the screening. Another possible reason for the decline was the development of the combinatorial chemistry, which first offered a simplest approach to obtain synthetic compound libraries with a relatively wide chemical diversity. This also might be explained partly because of new regulations enforced by the Rio convention on biodiversity, which restricted the screening of vast extract libraries ${ }^{[6]}$ and partly because traditional bioassay-guided fractionation techniques are generally regarded by the industry as too slow to fit into the pace of intensive high-throughput screening campaigns. ${ }^{[7]}$ However, the importance of NPs for human health is still crucial, and recent technological advances coupled with unrealised expectations from current lead-generation strategies have led to a renewed interest in NPs for drug discovery. ${ }^{[5]}$ The approaches used to study biologically significant NPs must therefore continue to evolve, and a new vision for natural medicinal agents must be found that embraces intellectual property, biology, chemistry, information systems, technology and biotechnology. ${ }^{[8]}$
In recent years, new approaches that are complementary to conventional bioactivity-guided fractionation, which is based on bioprospecting, have emerged for investigating bioactive NPs. One new approach of interest is reverse pharmacognosy, where a biological target is found using virtual or real screening. Results generated by this in silico approach might provide a good way to target pharmacophores and to select sustainable natural sources containing compounds holding these structural features for further isolation and bioactivity characterisation. ${ }^{[9]}$ Biotechnology approaches, such as metabolic engineering and synthetic biology, also offer new means to obtain NPs of interest using optimised biosynthetic pathways. ${ }^{[10]}$ In this respect, methods for activating silent biosynthetic pathways using stress induction or co-cultures of microorganisms, ${ }^{[11]}$ briefly described here, represent new routes to pursue. In addition, the development of highly informative biological screening methods that are compatible with assays on crude extracts represent a key point. In this respect, bioassays based on zebrafish are well suited for the development of rapid and efficient bioactivity-guided isolation methods that target new lead compounds with favourable activities and ADMET profiles. ${ }^{[12]}$ Such in vivo assays provide an attractive strategy to assess the in vivo effects of NPs at the microgram scale using microplates compatible with high- or medium-throughput screening. The development of analytical 
methods for the rapid identification of NPs in complex mixtures, such as plant extracts (dereplication), in parallel with approaches to profile the bioactivity simultaneously with HPLC separation, have considerably increased the pace at which bioactive NPs can be identified.

In this respect, our research unit has developed various strategies for the rapid identification of NPs (dereplication) that complement conventional bioactivityguided fractionation approaches. These strategies can be used for a comprehensive evaluation of the metabolite composition of natural extracts (metabolomics) and for the biological profiling of extracts at the microgram scale (microfractionation). Such approaches were applied to study poorly investigated natural sources of bioactive NPs, including plants and fungi, after induction of the chemical defence in these organisms by various stresses. Several of these aspects are discussed and illustrated in this paper.

\section{Induction of Chemical Defences}

\section{Stress Induction for Lead \\ Discovery}

Drug discovery programmes based on plant natural products have mainly exploited secondary metabolites, which often are constitutive defence metabolites. ${ }^{[13]}$ In classical lead discovery, the dynamic stress response of an organism is usually not considered, although the antimicrobial activity of many phytoalexins has been reported.[14]

Interestingly, minor compounds with strong bioactivities can be found by exploiting the dynamic defence response of plants by elicitation experiments. ${ }^{[15}$ Elicitors are often used to enhance the production of secondary metabolites in microorganisms; however, this strategy is limited mainly to the targeted production of given metabolites. ${ }^{[16]}$ In drug discovery, elicitors significantly affect the production of bioactive compounds in plants and dramatically increase the hit rate. ${ }^{[17]}$ Studies of antibacterial, antifungal and anticancer agents have shown that the number of plant extracts that give positive responses in these assays is more than doubled for elicited plants. For these reasons, plant metabolite induction and the exploitation of natural elicitation mechanisms in hostplant interactions may enhance the drug discovery hit rate when screening natural extracts.

\section{Search for Stress-induced Signalling Molecules in Plants}

To better understand how stress can induce chemical defences in plants and thus lead to the production of novel bioactive NPs of therapeutic or agrochemical interest, our research unit has performed fundamental studies on the early chemical events that trigger the wound-response in plants. In particular, as described below we have developed an original LC-MS-based metabolomics approach for the detection and localisation of minor but significant stress-induced biomarkers among many major, constitutively produced NPs. ${ }^{[18]}$ Besides the detection of known plant stress-hormones such as jasmonic acid, this non-targeted approach has provided a good way to discover new stress biomarkers (mainly oxylipins). A detailed study of the induction kinetics of these jasmonates has provided new insight into how plants rapidly perceive the stress caused by herbivores and how they then build up adapted chemical defences. ${ }^{[19]}$ Such an approach is currently pursued to study long-distance signalling in the model plant Arabidopsis thaliana and for the above- and belowground (leaves $v s$ roots) stress response in crop plants, such as maize (collaboration with E. Famer at University of Lausanne and T. Turlings at University of Neuchâtel).

\section{Confrontation of Microorganisms to Induce Silent Biosynthetic Pathways}

The exploitation of dynamic response phenomena can increase the likelihood of finding induced compounds with a new structure and enhanced bioactivities. This can thus represent an innovative way to search for new lead compounds from natural sources and to unravel some aspects of the complex, chemically mediated events that occur in the response to stress. Therefore, we have studied interspecies crosstalk to induce the production of new compounds through biosynthetic pathways encoded by genes that are often silent.[20]

In this respect, a Sinergia project supported by SNF (in collaboration with the Mycology Group (K. Gindro, Agroscope Changins-Wädenswil, ACW) and the Department of Dermatology (M. Monod, 'Centre hospitalier universitaire vaudois', CHUV)) has been initiated to study and exploit the natural biochemical induction that occurs in fungus-fungus and fungusplant interactions to search for new types of antifungals that are particularly effective against Fusarium species (spp.).[21] The study of Fusarium spp. is of great interest; besides being common fungal soil saprophytes, Fusarium spp. are frequently plant pathogens. In addition, these fungi have not only emerged as major opportunistic fungi in patients with severe immunosuppression, but they have also been found to be responsible for onychomycosis. Fusarium spp. are especially difficult infectious agents to combat, and the need is high for new antifungal drugs for both medicinal and agronomical use. This opens new research areas in the search for fungicidal natural products and the identification of Fusarium spp. traits related to human and plant pathogenicity. ${ }^{[21,22]}$

To produce novel metabolites with potent antifungal activities against these microorganisms, Fusarium spp. were co-cultivated on petri dishes with various other fungi and were then challenged with a broad range of microorganisms (an example of a confrontation between Trichophyton rubrum and Cladosporium sp. is shown in Fig. 2, below). As a result, four morphologically different confrontation-behaviours (interaction types) were observed and hundreds of fungal combinations were classified accordingly. The study of the confrontation zones using a metabolomics approach (see below) revealed a clear induction of various metabolites that could be assigned to a given 'combative' fungus. The biological activity of the induced fungal metabolites produced in this chemical warfare is currently studied in more detail. This investigation is also now extended to Fusarium plant interactions with both human and phypatogenic strains to identify antifungal plant stress constituents. Overall, the genetic, metabolic and behavioural observations made with these different Fusarium strains should provide a comprehensive list of common traits that might better explain the dual plant and human pathogenic nature of such microorganisms. Future larger screening efforts with other types of microorganisms, including $C$. albicans and $S$. cerevisiae, may also result in a better understanding of the mode of action of the identified fungicidal compounds and suggest methods to improve their activity profiles.

\section{Use of Other Types of Stresses to Elicit the Production of Bioactive Compounds}

In addition to studying the induction of bioactive natural products in plants and fungi due to biotic stresses, generic abiotic stress induction methods, such as UVc irradiation of plant leaves, have been considered as a generic technique to induce secondary metabolite production in plants. In this case also, change in metabolome composition between control and UV-stressed plants can be highlighted by differential metabolomics. In collaboration with ACW (O. Schumpp and K. Gindro), plants from very diverse origins were screened in this manner. A very strong induction of stilbenes could be measured in UV-irradiated leaves, such as those from Vitis spp. ${ }^{[23]}$ In addition, it has been also demonstrated that UV irradiation significantly enhanced antifungal activity. However, this effect seems to depend on the plant species, as in certain 


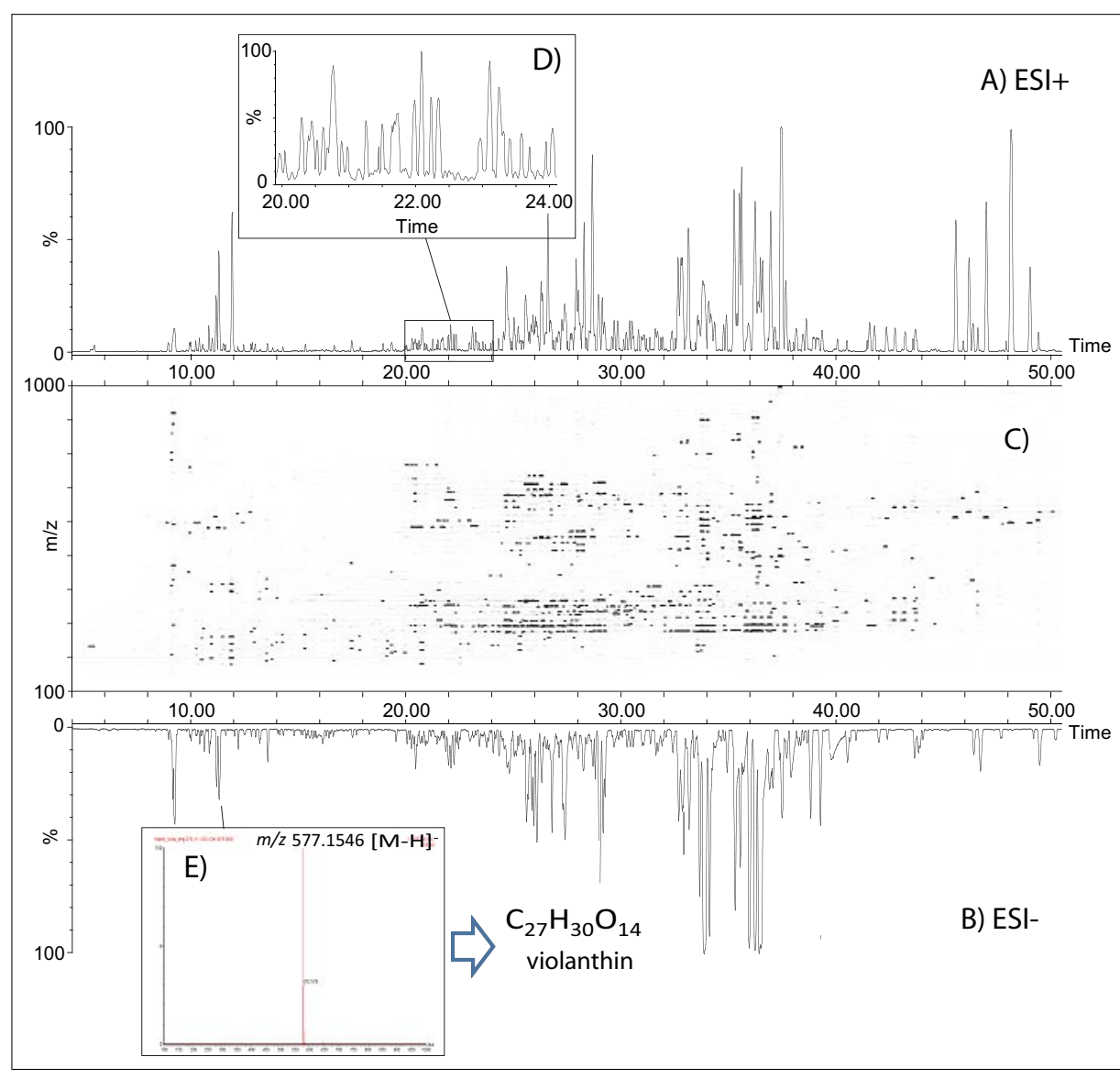

Fig. 1. UHPLC-TOF-MS profiling data of the $\mathrm{MeOH}$ extract of Viola tricolor using a generic gradient on a C18 UHPLC column $(150 \times 2.1 \mathrm{~mm}$ I.D.; $1.7 \mu \mathrm{m})$. A) Total ion current trace in positive ESI mode. B) TIC in negative ESI mode. C) 2D plot of the positive ESI ion map showing all the different ions detected in relation with their retention times ( $\mathrm{m} / \mathrm{z}$ vs RT). D) Inset showing the separation of minor constituents. E) Representative ESI HR TOF-MS of the flavonoid violanthin.

cases, UV treatment may sometimes lead to decreased fungitoxicity. ${ }^{[24]}$

All of these studies demonstrated that the induction of natural product production represented a potentially interesting approach for finding bioactive NPs that can either play a role in chemical ecology or be of strong interest for pharmaceutical and agrochemical lead discovery.

\section{Dereplication Profiling and Metabolomics}

To detect and identify stress-induced and bioactive NPs, efficient metabolomics approaches have to be developed to highlight the biomarkers of interest. These approaches should ideally enable rapid assessment of NP bioactivities at the analytical level. These methods are challenging to develop because they require high chromatographic resolution for detailed NP profiling as well as high throughput for rapid quantification or fingerprinting analyses. Furthermore, for dereplication purposes, these methods should also provide on-line spectroscopic information for the identification of each individual metabolite.
In this respect hyphenated techniques such as LC-MS, mainly, have played key roles over the last three decades. The reLiquid Chromatography (UHPLC) systems coupled to High-Resolution Time-OfFlight (TOF)-MS instruments (UHPLCTOF-MS) has represented an important breakthrough for the profiling of complex mixtures, such as natural extracts. ${ }^{[25]}$ In comparison to conventional HPLC, UHPLC allows a remarkable decrease in analysis time as well as an increase in peak capacity, sensitivity and reproducibility. [26] TOF-MS provides sensitive detection, high mass-resolution and high mass-accuracy for the rapid on-line assignment of molecular formulae of NPs: a key piece of information for metabolite identification and dereplication. In contrast, UHPLC-TOFMS provides very reproducible and highly informative datasets for MS-based metabolomics. ${ }^{[27]}$ In this context, our group has developed, in collaboration with S. Rudaz at UNIGE, generic methods for rapid fingerprinting and extensive high-resolution profiling of NPs in numerous extracts.

An example of the type of data generated by UHPLC-TOF-MS profiling for the cent introduction of Ultra-High Pressure analysis of a typical crude plant extract (Viola tricolor) is illustrated in Fig. 1. The upper and lower chromatograms recorded in positive (Fig. 1A) and negative ionisation modes (Fig. 1B), respectively, provide a rather comprehensive detection of metabolites that are present in an extract. The 2D plot in Fig. 1C represents the LC-MS data that can be used either for data mining of many samples using differential metabolomics or for dereplication, peak annotation and metabolite identification. Each dot (feature) in this plot is linked to a given metabolite, and (as shown, Fig. 1C) several hundreds of these features can be reproducibly detected to give a very detailed picture of the metabolome of any organism under study. As shown in the inset (Fig. 1D) UHPLC profiling enables the separation of a very high number of NPs and the detection of minor constituents. It has to be noted that, despites the high sensitivity of the method and since the MS response is compound dependant, LC-MS alone is not able to detect 'all' metabolites present in a given organism. The high mass accuracy data generated by TOF-MS in this type of profiling on the other hand provides molecular formula information for all NPs detected as shown for the flavonoid diglycoside violanthin (Fig. 1E).

MS-based metabolomics was very efficient for localising stress-induced compounds in fungal confrontation experiments in petri dishes (discussed above). In Fig. 2, an example is given of how induced fungal metabolites can be highlighted in a confrontation zone in a typical fungal co-culture. In this case, the extracts of different replicates of the pure culture of each fungus (Fig. 2A), as well as the extracts of the confrontation zone that had been precisely excised from the co-culture (Fig. 2B), were compared. UHPLC-TOFMS fingerprints were rapidly acquired for each extract. Representative ion maps of these fingerprints are displayed in Fig. 2C. Based on the mining of these datasets, a reconstructed ion map (calculated after automatic peak picking) showed ions characteristic of both fungi (Fig. 2D). This use of fingerprints provides a good way to highlight compounds only found in the confrontation zone but not in pure cultures (features marked in red in Fig. 2D). This procedure enabled the discrimination of stress-induced compounds (e.g. the mycoalexins) from constitutively produced compounds. As shown in Fig. 2E, some of the stress biomarkers are well-localised based on their single ion traces in the fingerprints. The generated TOF-MS data provided putative molecular formulae assignments (Fig. 2F).

With this type of information, and after scaling up the production of the fungal metabolites to a few dozen petri dishes, a 


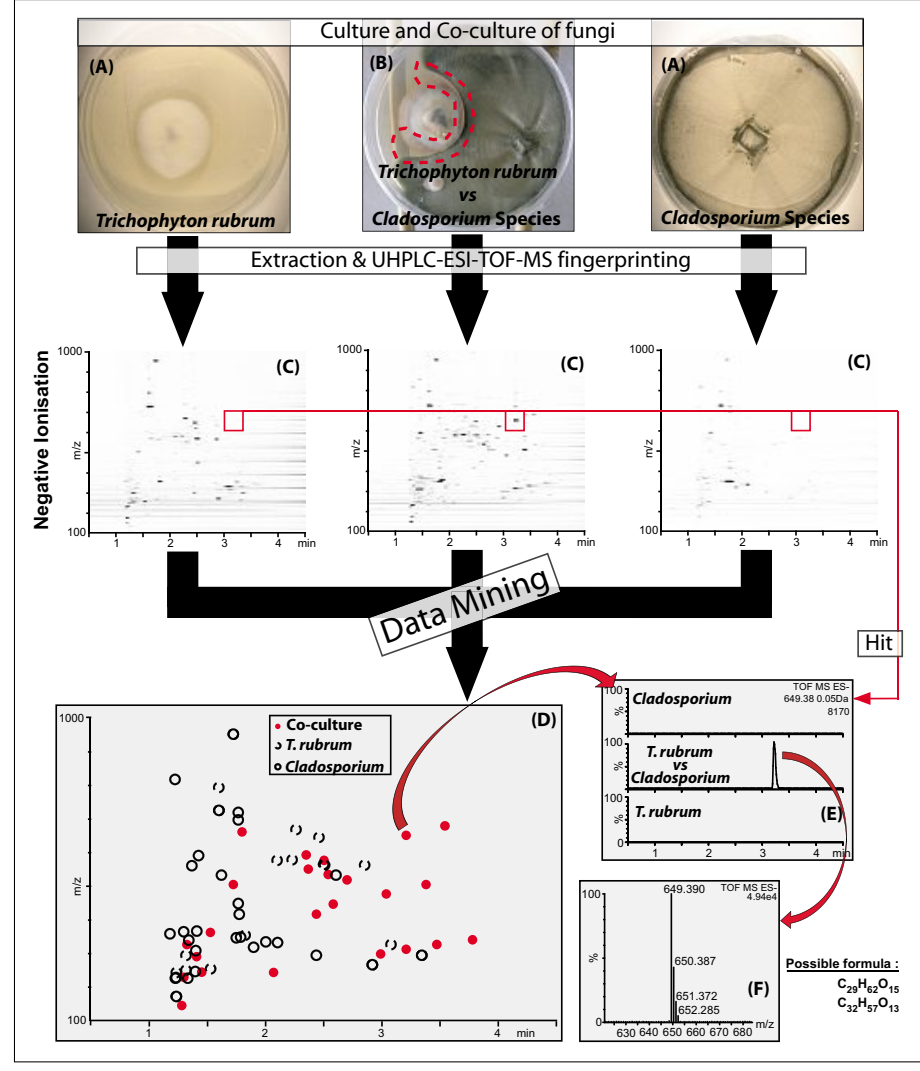

Fig. 2. General strategy for the study of metabolite induction by fungal cocultivation. A) Culture of pure strains of Trichophyton rubrum and Cladosporium $s p$. B) Co-culture of $T$. rubrum with Cladosporium sp. The red dashed zone was extracted for the profiling of the confrontation. C) UHPLC-ESITOF-MS ion maps of the crude extracts of each cultures. D) Localisation of induced metabolites only found in the confrontation zone (red dots) based on data mining. E) Extracted single ion traces of a specific stress biomarker present only in the confrontation zone. F) TOF-MS spectrum of the selected biomarker leading to molecular formula assignment. targeted micro-isolation procedure using semi-preparative LC-MS can be used for bioactivity assessment and complete structure elucidation of biomarkers. [28]

\section{Microfractionation, Biological Profiling and de novo Structure Elucidation}

To assess the bioactivity of any NP, either dynamically induced as described above or constitutively produced as for many herbal drugs, the compounds of interest must be purified. This step is also crucial for the de novo structure elucidation of unknown compounds by 1D and 2D NMR.

Conventional isolation approaches often employ relatively complex schemes that involve different preparative chromatographic methods requiring $\mathrm{kg}$ amounts of biological material to obtain $\mathrm{mg}$ amounts of pure NPs. Such approaches are effective and still important if a large amount of a given NP is needed for detailed studies of its mode of action. However, these approaches are rather time-consuming when performed in a non-targeted manner; they can also lead to NPs of low interest and with poor biological properties. With state-of-the-art bioassays and microNMR methods, the entire process can be performed at the $\mu \mathrm{g}$ level and the approach can be miniaturised. This considerably speeds up the identification of biomarkers and enables the preliminary assessment of their bioactivities.

To use this approach, a direct link has to be established between the analytical profiling of the crude active extract, the spectroscopic data obtained on-line and a few informative bioassays. Therefore, over the last decade, various HPLC-based methods have been developed for activity profiling as well as efficient isolation and subsequent structure elucidation. ${ }^{[25]}$ Our research unit has developed new strategies for biological and chemical screening to obtain valid chemical and bioactivity information prior to commencing large-scale isolation work (Fig. 3).

The strategy developed combines UHPLC-TOF-MS profiling followed by semi-prep HPLC-MS microfractionation of the natural active extracts in 96-well plates (Fig. 3). In a first screening step, the extracts from different origins are evaluated against different biological targets. The most promising active extracts are then analysed by the UHPLC-TOF-MS with generic $1 \%$ and $3 \%$ slope reversed phase gradients (Fig. 3A).[29] This metabolite profiling step gives a first idea on the complexity of the extract composition and preliminary information on the nature of their constituents. In order to rapidly localise the active ingredients, a simple linear gradient can be calculated from this profiling data. The separation is optimised for a semi-prep HPLC-MS fractionation in a maximum of 96 wells, which is a generic format for all of our biological assays. This enables the localisation of the chromatographic zone responsible for the biological activity (Fig. 3B). This rough separation usually does not directly yield pure compounds. A second microfractionation step can be efficiently performed on the

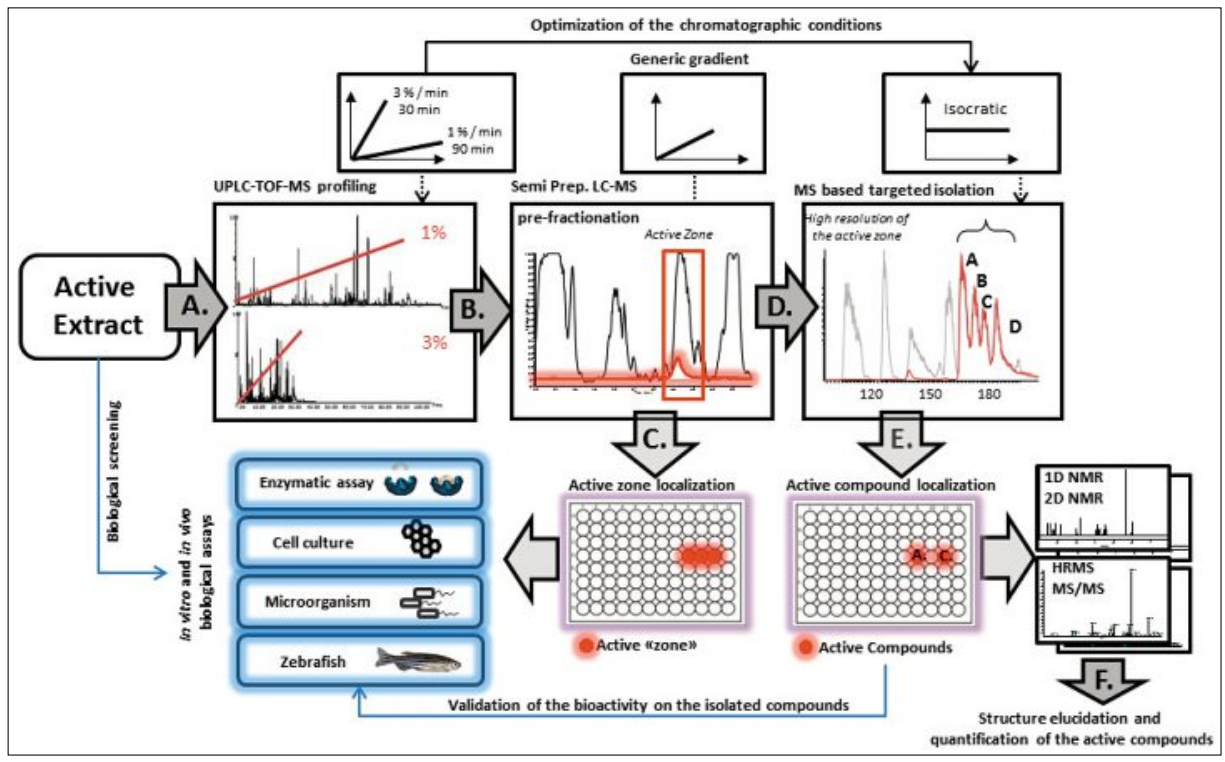

Fig. 3. Integrated platform for biological and chemical profiling of a natural active extract and targeted microfractionation for the identification of compound(s) of interest. A) Profiling with two generic gradients for separation optimisation using modelling software. B) Rapid fractionation of the crude extract using semi-preparative HPLC-MS C) In vitro and in vivo biological assays performed in 96 well-plates for the localisation of the compounds of interest. D) Final purification of microfractions of interest based on optimised conditions modelled from (A). E) Localisation of the bioactive constituents after the final purification step F) Purity assessment, structure elucidation and quantification at the $\mu \mathrm{g}$ level. 
active fraction based on the preliminary LC-MS information obtained. For this, the chromatographic conditions are calculated with the help of modelling software from the steep and slow gradient separations performed during the profiling step of crude extract (Fig. 3A). ${ }^{[29]}$ Since all profiling and isolation steps are monitored by MS, molecular ion species and retention time information obtained at the analytical level provides a good means to predict the chromatography conditions needed for this final purification of the bioactive NPs.

Finally, the zone holding the active constituents is microfractionated with high resolution and fully optimised LC conditions with this second semi-prep LC-MS chromatographic step to yield the pure compounds (Fig. 3D). The new 96-well plate is then submitted to the bioassay (Fig. 3E) and the active compounds are precisely localised. Micro-flow NMR used in parallel to this process on aliquots of the bioactive microfractions is used i) to evaluate their purity, ii) for the de-novo structure identification in complement to HR-MS since 1D and 2D NMR spectra can be recorded at the microgram level, ${ }^{[25]}$ iii) to estimate the sub-mg amount of bioactive compounds collected by absolute NMR quantification (Fig. 3F). ${ }^{[30]}$

The strategy proposed has been used in combination with several different assays at the enzyme (e.g. acetylcholinesterase and histone deacetylase inhibitors), cell culture (e.g. cytotoxicity and inhibition of tumorigenesis) and microorganism (e.g. Candida albicans, Fusarium spp.) levels. In addition, in vivo measurement can also be performed using the zebrafish model (e.g. anti-inflammatory and anti-epilepsy activities). For any given disease, a combination of different assays at different levels of complexity ranging from enzyme and cells to living organisms is important, and the possibility of performing all of these assays on a LC-peak from a natural active extract is a key element for early lead discovery. In particular, the zebrafish model that has recently emerged as an attractive in vivo system to study functional genomics drug discovery can also be used to assess toxicity. ${ }^{[30]}$ Thus, based on this miniaturised and integrated strategy, a relative comprehensive estimation of the bioactive potential of a given NP can be rapidly estimated. All bioassays represents a collaborative effort; the enzyme and cell assays are performed by P. A. Carrupt and M. Cuendet at UNIGE, and the zebrafish assay is conducted by P. De Witte and A. Crawford at the University of Leuven.

The whole process which is still presently under optimisation should provide a good mean to evaluate the potential of any NP in the early stages of the discovery process with limited amount of biological material.
Based on this approach, if a given NP is considered worthwhile to study indepth it can be isolated at large scale in a targeted manner. In this case, if it is a known compound, it might be isolated from a sustainable plant source available in large amount or by resupply of the plant or organism of interest. For this, the whole separation process can be optimised based on chromatographic calculation and gradient transfer. At this stage Medium Pressure Chromatography (MPLC) provides a good mean to yield mg amounts of NPs from gram amount of refined extracts. Efficient gradient transfer calculations to ensure the same selectivity of separation at both semipreparative HPLC and MPLC level from UHPLC are performed in collaboration (J-L. Veuthey, S. Rudaz and D. Guillarme, UNIGE).

\section{Conclusions}

In the drug discovery process, NPs still represent an important source of new pharmacophores, and the development of efficient dereplication protocols is essential for using a rational approach. The exploitation of NPs produced as stress biomarkers, by studying plants of fungi from a chemical-ecology perspective, represents a new way to discover interesting NPs for lead discovery because it involves the expression of metabolites from otherwise silent biosynthetic pathways. Further exploitation of these NPs may be achieved through biosynthetic chemistry or metabolic engineering approaches, which are emerging research fields in drug discovery. ${ }^{[31]}$ To broaden the diversity of NPs of therapeutic interest, new significant technology advancements provide an opportunity to explore minor constituents of new and sustainable sources of NPs that can yield new bioactivities after chemical or biological manipulations. Metabolome information obtained by generic profiling methods can also be used for reverse pharmacognosy approaches to efficiently target the isolation of in silico-identified compounds with high putative bioactivities. Our research unit is now pursuing investigations in this direction.

The combination of state-of-the-art analytical and spectroscopic methods and information-rich bioassays up to the in vivo level opens many new avenues for NP research. Screening of well-defined microfractions in 96-well plates avoids the difficulty of interpreting bioassay results obtained with crude extracts and the incompatibilities often encountered with such samples. The obtaining of micro-fractions also allows building up a semi-pure natural products library rapidly without the need of obtaining fully purified NPs in a first instance. This significantly speeds up the constitution of such libraries.

In addition, the development of a metabolomics method for finding stress metabolites provides a good way to target the isolation of biomarkers of interest solely based on the MS characteristics of the biomarkers. Metabolomics is presently also implemented for the analysis of animal and human fluids (collaboration with M. Cuendet, P. Christen and S. Rudaz at UNIGE) to investigate the mode of action of phytopreparations with proven clinical efficacies for which active ingredients have not been identified. Such an approach is expected to yield information about prodrug, synergy and the effects of drug metabolism, which are other important aspect of NP research on medicinal plants and phytotherapy.

\section{Acknowledgements}

J. L. W. is thankful to the Swiss National Science Foundation for the financial support to develop the miniaturised microfractionation platform and the metabolomics studies (Grant no. 205320-124667/1 and CRSII3_127187). J. L. W. is also grateful to the National Centre of Competence in Research (NCCR) Plant Survival, a research programme of the Swiss National Science Foundation for supporting the plant metabolomic platform. P. Eugster and $\mathrm{H}$. Kratou are acknowledged for providing Fig. 1, S. Bertrand, O. Schumpp and K. Gindro for Fig. 2.

Received: February 29, 2012

[1] J. Hong, Curr. Opin. Chem. Biol. 2011, 15, 350.

[2] D. J. Newman, G. M. Cragg, Future Medicinal Chemistry 2009, 1, 1415.

[3] J. Larsson, J. Gottfries, S. Muresan, A. Backlund, J. Nat. Prod. 2007, 70, 789.

[4] D. D. Baker, M. Chu, U. Oza, V. Rajgarhia, Nat. Prod. Rep. 2007, 24, 1225.

[5] F. E. Koehn, G. T. Carter, Nat. Rev. Drug Discovery 2005, 4, 206

[6] M. Kartal, Phytother. Res. 2007, 21, 113.

[7] D. G. I. Kingston, D. J. Newman, Idrugs 2005 , 8, 990.

[8] G. A. Cordell, M. D. Colvard, Arkivoc 2007, 97.

[9] Q. T. Do, C. Lamy, I. Renimel, N. Sauvan, P. Andre, F. Himbert, L. Morin-Allory, P. Bernard, Planta Med. 2007, 73, 1235.

[10] J. W. H. Li, J. C. Vederas, Science 2009, 325, 161.

[11] D. C. Oh, C. A. Kauffman, P. R. Jensen, W. Fenical, J. Nat. Prod. 2007, 70, 515.

[12] A. Crawford, C. Esguerra, P. de Witte, Planta Med. 2008, 624.

[13] K. Hostettmann, A. Marston, J. L. Wolfender, Chimia 2005, 59, 291.

[14] R. Hammerschmidt, Annu. Rev. Phytopathol. 1999, 37, 285.

[15] A. Vasconsuelo, R. Boland, Plant Sci. 2007, 172,861 .

[16] S. Sanchez, A. L. Demain, Enzyme Microb. Technol. 2002, 31, 895.

[17] A. Poulev, J. M. O’Neal, S. Logendra, R. B Pouleva, V. Timeva, A. S. Garvey, D. Gleba, I. S. Jenkins, B. T. Halpern, R. Kneer, G. M. Cragg, I. Raskin, J. Med. Chem. 2003, 46, 2542.

[18] E. Grata, J. Boccard, D. Guillarme, G. Glauser, 
P.-A. Carrupt, E. E. Farmer, J.-L. Wolfender, S. Rudaz, J. Chromatogr. B 2008, 871, 261.

[19] G. Glauser, E. Grata, L. Dubugnon, S. Rudaz, E. Farmer, J. L. Wolfender, J. Biol. Chem. 2008, $283,16400$.

[20] G. Glauser, K. Gindro, J. Fringeli, J.-P. de Joffrey, S. Rudaz, J.-L. Wolfender, J. Agric. Food Chem. 2009, 57, 1127.

[21] S. Schurch, K. Gindro, O. Schumpp, M. Monod, J. Verrier, N. Bohni, J.-L. Wolfender, Agrarforschung Schweiz 2010, 1, 442.

[22] H. L. Mehl, L. Epstein, Environ. Microbiol. 2007, 9, 2189.
[23] G. Marti, J. Boccard, S. Rudaz, K. Gindro, J. L. Wolfender, Chimia 2011, 65, 481.

[24] O. Schumpp, N. Bruderhöfer, J. Monod, J. L. Wolfender, K. Gindro, Mycoses 2012, submitted.

[25] J. L. Wolfender, G. Marti, E. F. Queiroz, Curr. Org. Chem. 2010, 14, 1808 .

[26] P. Eugster, D. Guillarme, S. Rudaz, J. L. Veuthey, P. A. Carrupt, J. L. Wolfender, J. AOAC Int. JAOAC 2011, 94, 51.

[27] J. L. Wolfender, G. Glauser, J. Boccard, S. Rudaz, Nat. Prod. Commun. 2009, 4, 1417.
[28] S. Bertrand, O. Schumpp, N. Bohni, M. Monad, K. Gindro, J. Wolfender, Planta Med. 2011, 77 , 1234.

[29] G. Glauser, D. Guillarme, E. Grata, J. Boccard, A. Thiocone, P.-A. Carrupt, J.-L. Veuthey, S. Rudaz, J.-L. Wolfender, J. Chromatogr. A 2008, $1180,90$.

[30] A. Crawford, N. Bohni, J. Maes, A. Kamuhabwa, M. Moshi, C. Esguerra, P. de Witte, J. Wolfender, Planta Med. 2010, 76 , 1331.

[31] J. D. Keasling, Science 2010, 330, 1355. 